\title{
Evidence for biological roots in the transgenerational transmission of intimate partner violence
}

\author{
MI Cordero ${ }^{1,2}$, GL Poirier ${ }^{1}$, C Marquez ${ }^{1}$, V Veenit ${ }^{1}$, X Fontana ${ }^{1}$, B Salehi ${ }^{1}$, F Ansermet ${ }^{2}$ and C Sandi ${ }^{1}$
}

Intimate partner violence is a ubiquitous and devastating phenomenon for which effective interventions and a clear etiological understanding are still lacking. A major risk factor for violence perpetration is childhood exposure to violence, prompting the proposal that social learning is a major contributor to the transgenerational transmission of violence. Using an animal model devoid of human cultural factors, we showed that male rats became highly aggressive against their female partners as adults after exposure to non-social stressful experiences in their youth. Their offspring also showed increased aggression toward females in the absence of postnatal father-offspring interaction or any other exposure to violence. Both the females that cohabited with the stressed males and those that cohabited with their male offspring showed behavioral (including anxiety- and depression-like behaviors), physiological (decreased body weight and basal corticosterone levels) and neurobiological symptoms (increased activity in dorsal raphe serotonergic neurons in response to an unfamiliar male) resembling the alterations described in abused and depressed women. With the caution required when translating animal work to humans, our findings extend current psychosocial explanations of the transgenerational transmission of intimate partner violence by strongly suggesting an important role for biological factors.

Translational Psychiatry (2012) 2, e106; doi:10.1038/tp.2012.32; published online 24 April 2012

\section{Introduction}

Intimate partner violence, particularly against women, is a pervasive worldwide phenomenon with serious societal and public health consequences for individuals, families, communities and societies. ${ }^{1,2}$ Despite increasing efforts to develop prevention and intervention programs that address intimate partner violence, evidence of positive outcomes is scarce because of an insufficient understanding of its causes., Although the roots of partner violence may be complex, childhood exposure to violence, either as a victim or a witness, has been identified as a major risk factor for intimate violence perpetration. 4,5 To date, the prevailing etiological theories proposed to explain intimate partner violence have emphasized two prominent factors: (i) the cultural environment, particularly the impact of male dominance in society (Cultural theory), or (ii) social learning, whereby children learn patterns of violence by watching relevant early figures - parents in particular-that are reproduced in intimate partner relationships in adulthood, which enables violence to be transmitted from one generation to the next (Social learning theory). Despite increasing evidence that early exposure to stress can affect the neurodevelopmental trajectories that influence an individual's behavior in adulthood, ${ }^{6-8}$ whether biological factors can account for the intergenerational transmission of intimate partner violence, both within an individual lifetime and across generations, remains under-researched. ${ }^{9}$ Most of the studies directed at identifying risk factors at the biological level are cross-sectional studies focused mainly on demographics, live stressful experiences, ${ }^{10,11}$ mental disorders ${ }^{12,13}$ and substance abuse, ${ }^{14,15}$ remaining unclear whether those biological factors are the cause or correlated factors of the violence perpetrated by the abusive partner. ${ }^{1,9}$

To seek evidence for the contribution of biological factors to the intergenerational transmission of intimate partner violence, we selected an animal model (that is, devoid of human cultural connotations) whereby exposing male rats to fear-inducing experiences from juvenility to puberty (that is, the peripubertal period ranging from postnatal days P28P42; subsequently referred to as peripubertally stressed rats) was shown to produce pathological aggression against males during adulthood. ${ }^{16}$ In this model, fear was induced in a non-social context, which allowed the exclusion of potential vicarious social learning. To determine whether peripubertal stress would result in abnormal aggressive behavior against a female partner, males from both the stress and the control groups were individually paired with a female and left to cohabit in a home cage for 21 days. Furthermore, we sought to evaluate whether biological factors could also account for the transfer of a behavioral profile of pathological aggression to an intimate partner across generations. To this end, we investigated whether the male offspring of peripubertally stressed males displayed increased aggression toward a female partner.

\section{Materials and methods}

Animals. The male experimental subjects were the offspring (F0) of Wistar Han rats purchased from Charles River

\footnotetext{
${ }^{1}$ Laboratory of Behavioral Genetics, Brain Mind Institute, School of Life Sciences, Ecole Polytechnique Fédérale de Lausanne (EPFL), Geneva, CH, Switzerland and ${ }^{2}$ Child and Adolescent Service of Psychiatry (SPEA), Hospital University of Geneva, Geneva, CH, Switzerland

Correspondence: Professor C Sandi, Laboratory of Behavioral Genetics, Brain Mind Institute, School of Life Sciences, Ecole Polytechnique Federale de Lausanne (EPFL), Lausanne, CH 1015, Switzerland.

E-mail: carmen.sandi@epfl.ch
}

Keywords: early life; epigenetics; psychopathology; stress; transgenerational transmission; violence

Received 19 December 2011; revised 24 February 2012; accepted 26 March 2012 
Laboratories (Lyon, France) that were bred in our animal house, as well as the offspring (F1) from F0 male and F0 females. The female experimental subjects were virgin Wistar rats acquired at 10-week-old from Charles River Laboratories. Animals were maintained under controlled conditions (12 h light/dark cycle; lights on at 0700 hours; $22 \pm 2{ }^{\circ} \mathrm{C}$ ). Food and water were available ad libitum. With the exception of animals submitted to peripubertal stress (see procedure below), all animals were at least 3-month-old at the beginning of the experiments. With the exception of the home cage interactions and maternal behavior observations (see details below), animal testing occurred during the first half of the animals' light phase. All conducted procedures conformed to Swiss National Institutional Guidelines on Animal Experimentation and were approved through a license by the Swiss Cantonal Veterinary Office Committee for Animal Experimentation.

F0 male rats. F0 male subjects were the male offspring of rats obtained from Charles River Laboratories. Male offspring from different litters were weaned at postnatal day 21. Rats from different litters were mixed and housed with 2-3 animals per standard plastic cage; an equivalent number of animals from each litter were randomly placed in the peripubertal stress and control groups ( $N=22$ per group).

Peripubertal stress applied to FO males. We followed a previously described peripubertal stress protocol. ${ }^{16,17}$ This protocol involves two different fear-inducing stressors: (i) the synthetic fox odor trimethylthiazoline (Phero Tech, Delta, BC, Canada) is a synthetic version of a compound found in fox feces that evokes innate fear in rats. A small amount of trimethylthiazoline $(9 \mu \mathrm{l})$ was absorbed onto a small cloth and administered in a white plastic box $(38 \times 27.5 \times 31 \mathrm{~cm})$ placed under a bright light (200-250lux) and (ii) exposure to an elevated platform $(12 \times 12 \mathrm{~cm}$, elevated $95 \mathrm{~cm}$ from the ground) under direct bright light (500-550lux). This is a stressful experience for rats because of their innate fear of height and open spaces. Each of these stressors lasted $25 \mathrm{~min}$ and was applied in a semi-random order. Following each stress session, the animals were returned to their home cages. During the first $15 \mathrm{~min}$, a transparent Plexiglas (MSPLAST, Pampigny, Switzerland) wall with holes separated each of the animals in the cage. The stressors were applied during the peripuberty period on an unpredictable schedule (a total of 7 days across P28-P42; on P28-30, P34, P36 and P40-P42) during the light phase. Stressor order and timing were changed on different days. On some stress days, only one stressor was presented; on others both were given consecutively. The control animals were handled on the days that their experimental counterparts were exposed to stress.

Fo female partners. Adult (10-week-old) virgin female Wistar rats (Charles River Laboratories) were used as experimental subjects. Upon arrival, female rats were housed in groups of three per cage (see experimental protocol in Figure 1a). Two weeks later, they were caged with a 3-month-old F0 adult male (either control or peripubertally stressed, $N=22$ per gender per group) for 21 days. At the end of this period, the male was removed before parturition and tissue paper was provided to facilitate nest building. Dams and offspring were not disturbed until P7, when the first cage change after delivery was performed. With the exception of weekly cage changing, litters and dams remained undisturbed until weaning on P21. Two days later, the females were again housed in groups of three (with their original cagemates) and kept under these housing conditions during the subsequent behavioral testing phase. One week after female re-housing, all females were submitted to a battery of behavioral tests that lasted 6-7 weeks (Figure 1b; control $N=10$; stress, $N=7$ ).

F1 male rats. Weaned male offspring of F0 males and females were reared in groups of three of same sex. Rats from different litters within the same experimental group (F1 control $N=13$; F1 stress $N=11$ ) were mixed in the respective home cages. Animals were left undisturbed until adulthood (three-month-old).

F1 female partners. The females that were caged with $F$ 1 male rats at the age of 3 months were the offspring of F0 male and F0 female controls. Once weaned, these females were reared in groups of 3 rats per cage. Rats from different litters were mixed. They were left undisturbed until adulthood (3-month-old), at which time they were individually caged with an F1 adult male (either control or the offspring of stressed F0 male rats, $N=11-13$ per gender per group) for 21 days. All other procedures were the same as described above for F0 female partners. Behavioral testing was carried out in $N=13$ controls and $N=11 \mathrm{~F} 1$ female stress rats.

Behavioral observations during male-female cohabitation. During male-female cohabitation, the home cage was changed three times (once per week) at $\sim 1700-1900$ hours. This arousing experience stimulates social interactions, and for this reason it was used as the starting time for behavioral observations. The first time each male and female were put together in the home cage, and the periods immediately following home cage changing on days 7 and 14 were video-recorded for $30 \mathrm{~min}$. The number of attacks from the male to the female and the time during which the female displayed defensive-submissive behavior (either freezing or being in a supine position under the male) were scored by an experimenter who was blinded to the treatment conditions and assisted by a computer program (The Observer 5.0.25, Noldus, Wageningen, The Netherlands, 2003). Animals were not visibly wounded by these behaviors, except for superficial scars on a few females.

Observations of maternal behavior. Maternal care for the first 6 days postpartum for each dam was observed using a protocol adapted from Champagne et al. ${ }^{18}$ Detailed methods are provided in the Supplementary Material.

Behavioral characterization of female emotionality. The long-term emotional effects of 21-day cohabitation with male partners were assessed 10 days after weaning using a battery of tests in the following order: elevated plus maze, open field (OF) and novel object reactivity test, forced swim test (FST), 
a

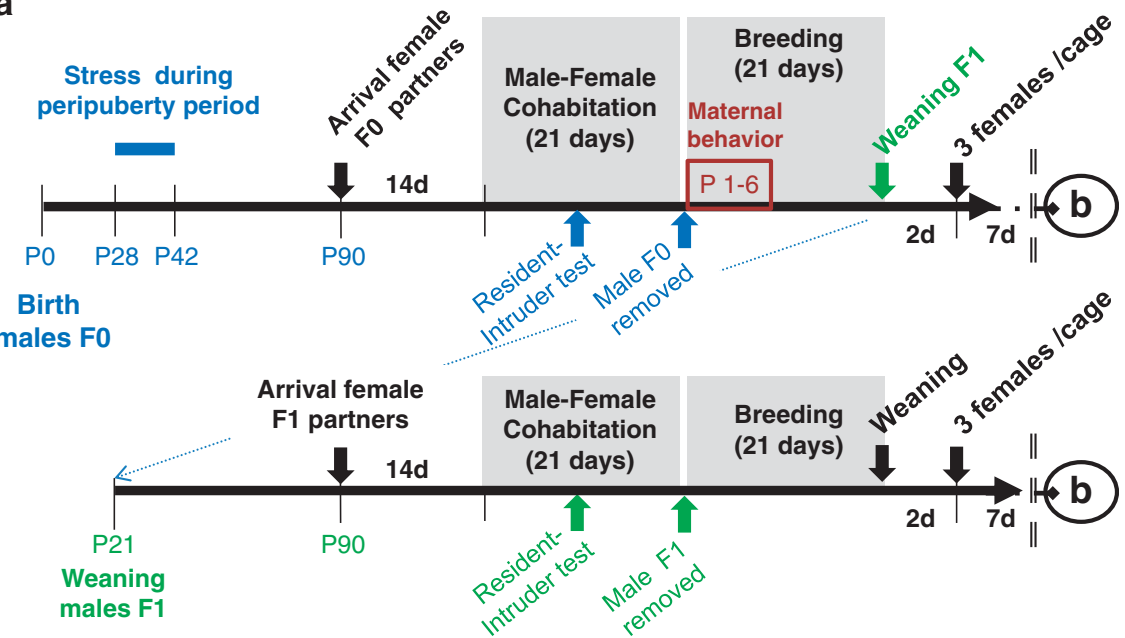

b

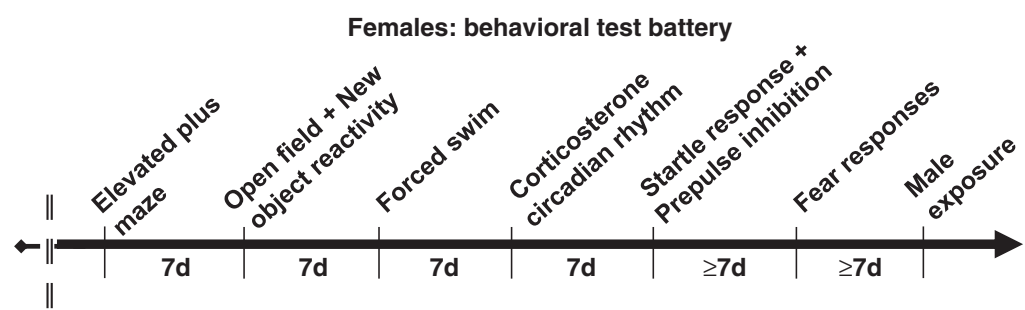

Figure 1 Schematic of the experimental procedures, including the general experimental design (a) and the sequence of behavioral tests performed in the females (b). Abbreviations: $d$, days; P, postnatal days.

startle response, prepulse inhibition (PPI) and fear induction experiences (shock-induced in F0 and male-induced in F1). There were at least 7 days between each test, with the total emotional testing period lasting 6-7 weeks. All behavioral testing was conducted between 0900 and 1400 hours. Except for the fear induction, shock-induced and the startle and PPI that were recorded automatically by software programs (see below), behavior was monitored using a video camera located on the ceiling, and movements of the rats were automatically registered and analyzed with a computerized tracking system (Ethovision 3.1.16, Noldus IT). Detailed methods are provided in the Supplementary Material.

Exposure to unfamiliar male as a challenge for neurobiological analyses. All F1 female partners reported in the F1 study and a group of F0 female partners were generated under equal conditions as reported for the FO study, except for the last behavioral test of fear induction to shock. In order to avoid potential interference of shockinduced learned helplessness with the neurobiological mechanisms investigated (see below ${ }^{19}$ ), the females of this study were exposed to a naïve unfamiliar male while in diestrus phase and before killing. For this test, each group of females was randomly divided into two conditions: basal (home cage control condition, $N=4-5$ per group) or exposure to an unfamiliar male (male exposure condition, $N=6-8$ per group). In the male exposure condition, the females were placed in a fresh cage containing a transparent Plexiglas separator that divided the cage into two compartments $(20 \mathrm{~L} \times 12 \mathrm{~W} \times 35 \mathrm{Hcm})$. The small size of these compartments ensured proximity and exposure of the experimental animal to the male stimulus. After a 5-min habituation period, an unknown sexually inexperienced 3month-old male was placed in the other compartment. The separator contained many small holes allowing visual, auditory and olfactory stimuli to pass between the chambers. After $15 \mathrm{~min}$, the females were returned to their home cage until they were killed (see details below in Brain processing and sectioning section).

Plasma corticosterone. To assess possible alterations of corticosterone circadian rhythm as a consequence of malefemale cohabitation with different types of males, blood samples were obtained from all females at two time points (morning, between 0800 and 0900 hours; and night, between 2000 and 2100 hours), 1 week after the FST (see experimental schedule in Figure 1b). Blood samples were collected by tail nick, which consisted of gently wrapping the animals with a cloth, making a 2-mm incision at the end of one of the tail arteries and then massaging the tail while collecting, within $2 \mathrm{~min}, 300 \mu \mathrm{l}$ of blood into ice-cold, heparin capillary tubes (Microvett cb300, Sarstedt, Sevelen, Switzerland). The tubes were centrifuged at $4{ }^{\circ} \mathrm{C}$ (4500 r.p.m., $20 \mathrm{~min}$ ), and plasma aliquots were stored at $-20{ }^{\circ} \mathrm{C}$ until the assay was performed. Because samples were collected on the same day (morning and night), the second collection was obtained by gently removing the scab from the first incision. 
Determination of estrous cycle phase. The estrous cycle was monitored in all females during the 3 weeks before the unfamiliar male challenge for neurobiological analyses (see above). Vaginal swabs were taken daily in all rats. Female rats were lightly restrained manually and a swab wet with room temperature normal saline solution $(\mathrm{NaCl} 0.9 \%$ ) was gently and shallowly inserted into the vagina and smeared on a new slide. The slide was immediately fixed with $1 \%$ toluidine blue solution and observed under the microscope ( $\times 10$ and $\times 40$ objective lenses) to evaluate cells that characterize each stage of the estrous cycle (for details on the procedure, see $\mathrm{Mandl}^{20}$ ).

\section{Tissue processing for immunohistochemistry}

Brain processing and sectioning. Two hours after onset of exposure to the male stimulus (see Exposure to unfamiliar male as a challenge for neurobiological analyses) or at an equivalent time for the home cage group taken directly from their home cages, the females were anesthetized with intraperitoneal injections of sodium pentobarbital and intracardially perfused with phosphate-buffered saline (PBS, $0.1 \mathrm{M})$ followed by $4 \%$ paraformaldehyde in PBS $(\mathrm{pH} 7.4)$ before brain removal. Whole brains were incubated in $30 \%$ sucrose until saturation and then flash-frozen in isopentane on dry ice and kept at $-80^{\circ} \mathrm{C}$ until processing. A cryostat (Leica, CM 3050 S, Heerbrugg, Switzerland) was used to cut $30-\mu \mathrm{m}$ sections that were stored as six alternate sets. Slices were been kept in cryoprotectant solution (glycerol, ethylene glycol in PBS) at $-20^{\circ} \mathrm{C}$ until immunohistochemistry was performed.

Double-immunostaining of c-Fos and serotonin (5-HT). A set of brain sections (usually a dozen per subject) including dorsal raphe nucleus (DRN; from $-7.30 \mathrm{~mm}$ to $-9.30 \mathrm{~mm}$ from the bregma ${ }^{21}$ was processed sequentially for immunohistochemical labeling of c-Fos and 5-HT. All tissues were processed with same solutions. Although multiple baskets were necessary to process all the slices, tissue from different groups was counterbalanced among the baskets to reduce potential immunohistochemistry lot artifacts. Sections were washed $(4 \times 10 \mathrm{~min}$ in PBS, $\mathrm{pH}$ 7.4), and then endogenous peroxidase was blocked by incubation in a solution consisting of 1 volume of $30 \% \mathrm{H}_{2} \mathrm{O}_{2}$, 1 volume of methanol, and 8 volumes of $0.1 \mathrm{M}$ PBS with Triton-X100 (PBST; Sigma-Aldrich, Buchs SG, Switzerland) for $10 \mathrm{~min}$ and then washed again. Next, the sections were incubated for $60 \mathrm{~min}$ in blocking buffer (5\% skim milk, 5\% normal serum and $0.01 \%$ sodium azide in PBST), followed by a wash before incubation in anti-c-Fos antibody in PBST (1:6000, sc-52, Santa Cruz Biotechnology, Heidelberg, Germany) for $48 \mathrm{~h}$ at $4{ }^{\circ} \mathrm{C}$. Sections were then washed again before incubation in secondary antibody with normal serum for $2 \mathrm{~h}$ at room temperature (Elite Vector Kit, rabbit IgG, Vector Labs, Peterborough, UK). After another wash, brains slices were incubated for $1 \mathrm{~h}$ with avidin-biotinperoxidase complex (Elite ABC Vector kit, Vector Labs) at room temperature. Sections were washed once more and finally rinsed in $0.05 \mathrm{M}$ Tris buffer $(\mathrm{pH} 7.4)$. Final staining was obtained using 3,3'-diaminobenzidine (DAB) chromogen with nickel enhancement (as per manufacturer's instructions;
DAB substrate kit, Vector Labs) and washing afterwards with cold PBS to stop the reaction. The sections were then labeled for 5-HT in a similar fashion but without the blocking steps. The primary antibody solution used consisted of anti$5 \mathrm{HT}$ antibody in PBST (1:2000, S5545, Sigma-Aldrich), which was visualized with SG chromogen (SG Substrate Kit, Vector Labs). The sections were mounted on microscope slides, dehydrated with a series of graded alcohols, cleared with xylene and coverslipped.

Double-labeled cell counting. Sections were viewed with an Olympus BX51 microscope (Olympus, Lausanne, Switzerland), the DRN and its subregions were identified, ${ }^{21}$ and double-labeled cells for c-Fos and $5-\mathrm{HT}$ were assessed by an experimenter blind to the treatment groups. Images were acquired using a ColorView Camera $(\times 10)$ (Olympus) and stored digitally. The cell counts were normalized to the area size.

Statistical analyses. Statistical analyses were performed with SPSS version 14.0 (SPSS, Chicago, IL, USA). Normality and homogeneity of variance were tested and adjusted statistics were used if required. Parametric statistics were applied when data were normally distributed according to the Shapiro-Wilk test. Mean comparisons were carried out with either Student's $t$-tests or analyses of variance (either oneway or factorial analyses of variances with or without repeated measures) as appropriate. Sphericity assumed modeling, with Greenhouse-Geisser $(\varepsilon<0.075)$ and Huynh-Feldt $(\varepsilon>0.075)$ adjustments, was applied. Simple main effect analyses and post hoc comparisons were made with Fisher's least significant difference test, as appropriate. When the data were not normally distributed even after square root or log 10 transformations, non-parametric analyses were carried out with the Mann-Whitney tests. If Levene's test for equality of variances was significant, equal variances were not assumed, and the altered degrees of freedom were rounded to the nearest whole number. Correlational analyses between the psychobiological measures obtained in the females were performed using the Pearson's correlation test. Values in graphs represent the mean \pm s.e.m. Sample sizes are indicated in figure legends. The results were considered statistically significant if $P<0.05\left(^{\star}\right), 0.01\left(^{\star \star}\right)$ or $0.001\left(^{\star \star \star}\right)$.

\section{Results}

Effects of peripuberty stress on males' aggressive behavior against females at adulthood. Analyses of male-female interactions indicated that peripubertally stressed males were more aggressive with females than the control males (Figure 2a), whereas females cohabiting with peripubertally stressed males showed a higher defensive-submissive behavior than those living with the control males (Figure 2b; see Supplementary Tables S3-S5, for a detailed statistical information).

Physiological and behavioral impact of cohabitation with the peripubertally stressed males on the female rats. To understand the physiological and behavioral impact 


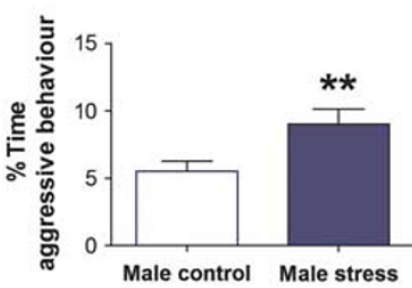

b

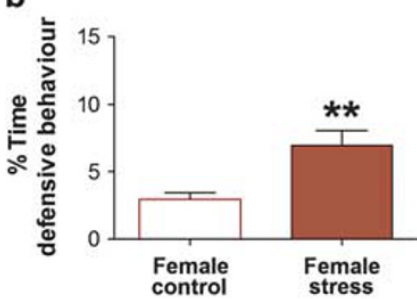

F0
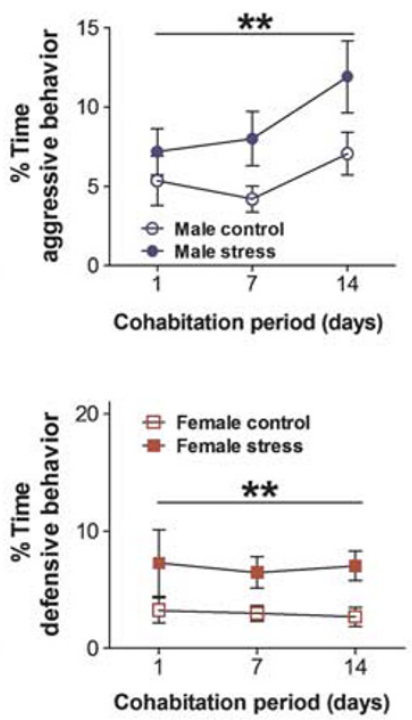

C

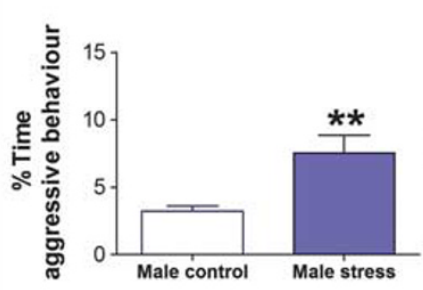

d

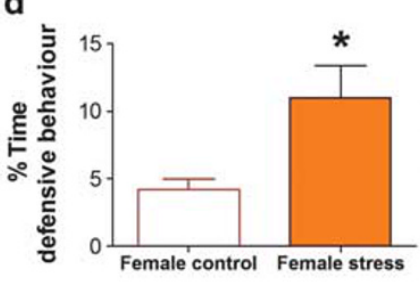

$\mathrm{F} 1$
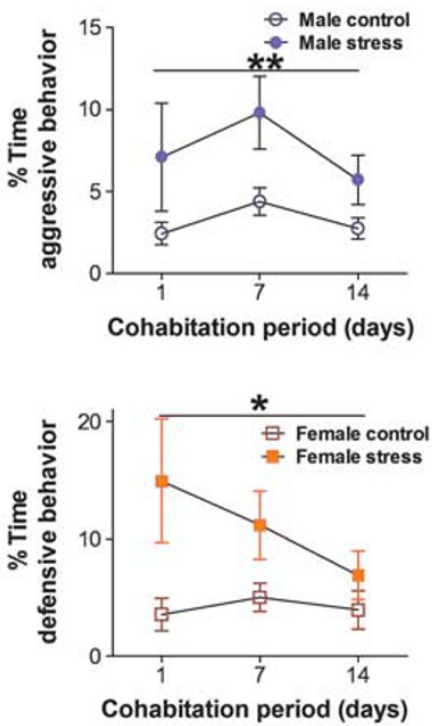

Figure 2 Aggressive and defensive behaviors during male-female cohabitation. During the 21 days of male-female cohabitation between pairs of rats involving either control or peripubertally stressed males (F0; $N=22$ per group) or control or male offspring from peripubertally stressed males ( $F 1 ; N=11-13$ per group), interactions were recorded weekly for 30 min beginning on the first day. ( $\mathbf{a}$ and $\mathbf{b})$ Males' aggressive behavior toward their female partners as the total average (left panel) and across weekly observations (right panel). The peripubertally stressed males (F0) and their male offspring (F1) show higher aggression levels than the control males. (c and d) Females' defensive behavior toward their male partner as the total average (left panel) and across weekly observations (right panel). Females (female stress) cohabiting with either a peripubertally stressed male or a male offspring from stressed fathers displayed a higher defensive behavior than those paired with a control male (female control). The results are the mean \pm s.e.m. ${ }^{*} P<0.05 ;{ }^{*} P<0.01$ versus control group.

of cohabitation with the peripubertally stressed males on the female rats, we monitored the females' body weights and indexes of activity in the hypothalamus-pituitary-adrenal axis as well as their behavioral responses to tests for anxiety- and depression-like behavior following cohabitation. After weaning, females were caged in groups of three from the same experimental group to avoid isolation stress while investigating the long-term impact of cohabitation with an aggressive male. Female partners of the peripubertally stressed males showed decreased body weights both during and up to 10 weeks after cohabitation (Supplementary Figure S1A, B, Supplementary Table S29). These females also showed an altered hypothalamus-pituitaryadrenal axis function, as indicated by alterations in the circadian pattern of plasma corticosterone levels monitored 7 weeks after cohabitation (Figure 3a, Supplementary Table S10) and by enlarged adrenal glands relative to the control females (Supplementary Table S1). At the behavioral level, females formerly partnered with the peripubertally stressed males (F0) showed the following behaviors: (i) enhanced anxiety-like behavior in the elevated plus maze (Figure $3 \mathrm{~b}$, Supplementary Table S11) and startle responses (Figure 3d, Supplementary Tables S19, S20); (ii) increased motor reactivity both in an OF and in response to a novel object (Figure 3c, Supplementary Tables S13, S15, S17, S18); (iii) increased PPI (Figure 3e, Supplementary Table S21); (iv) enhanced depression-like behavior in the FST (Figure 3f, Supplementary Table S22) and (v) enhanced fear responses to mild aversive stimulation (Figure $3 \mathrm{~g}$, Supplementary Table S23).
Increased aggression toward females in the male offspring of peripubertally stressed males. We sought to evaluate whether biological factors could also account for the transfer of a behavioral profile of pathological aggression to an intimate partner across generations. To this end, we investigated whether the male offspring of peripubertally stressed males displayed increased aggression toward a female partner, similar to their fathers, and examined the physiological and behavioral consequences of this interaction for their female partners. Overall, the group of all female partners of peripubertally stressed males presented obstetric problems, cannibalism and altered maternal care (Figure 4, Supplementary Tables S2, S30). However, importantly, the violence transmission part of the study was performed in offspring whose maternal care did not seem to differ from the one displayed by control females [(Figure 4, Supplementary Figure S2, Supplementary Table S30); maternal behavior was scored in the dams' home cage during the first 6 days postpartum according to Champagne et al. $^{18}{ }^{18}$ ] as only the male offspring (two males per litter were selected for the follow-up study, representing the average body weight of the litter) of females not displaying obstetric problems or cannibalism were used for the F1 study. Thus, we pursued an experimental design equivalent to the design followed for the previous generation (that is 21 days of male-female cohabitation, with males from either control or peripubertally stressed fathers and females always from control rats, followed by a behavioral and physiological assessment of the females). Strikingly, the results showed that the male offspring, without postnatal contact with their fathers, reproduced the response pattern of their 

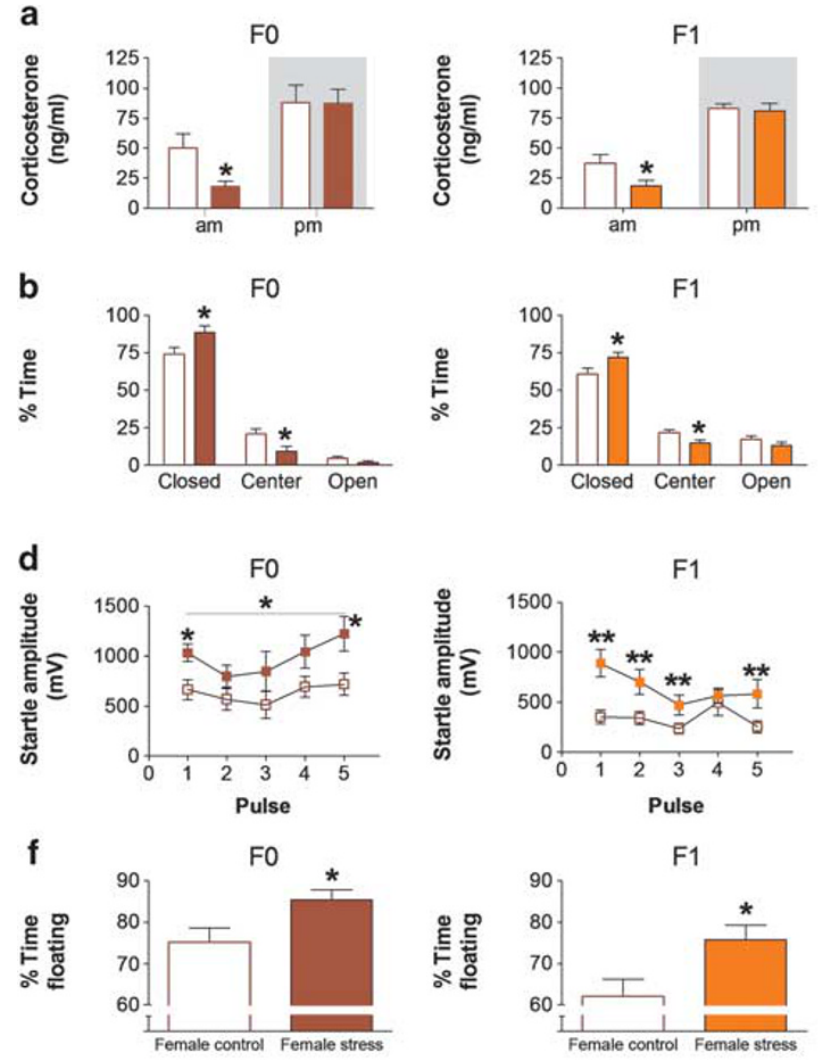
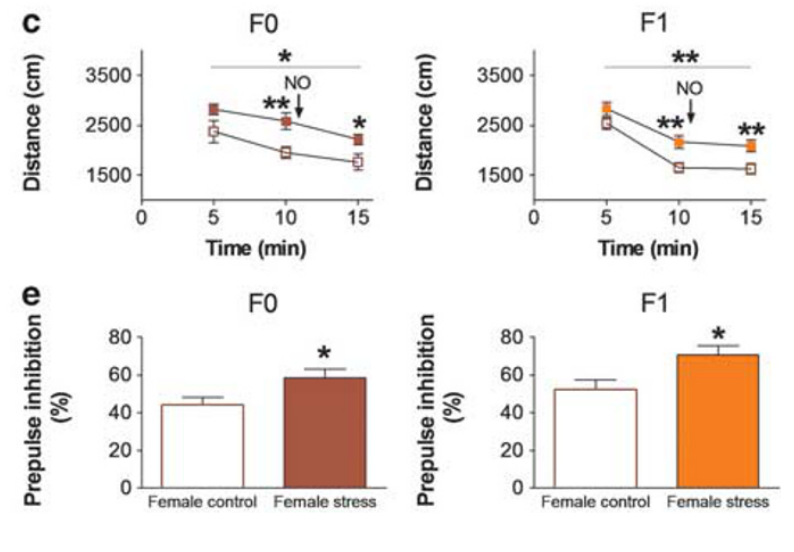

Female control

Female stress F0

Female stress F1

Figure 3 Long-term physiological and behavioral impact of male-female cohabitation on females. (a) Circadian plasma corticosterone levels evaluated at 7 weeks after cohabitation. Females that had cohabited with either a peripubertally stressed male (F0) or a male offspring from stressed fathers (F1) had lower morning corticosterone levels than controls. In behavioral tests of emotionality, compared with the control females, rats in the Female stress groups showed the following behaviors: (b) increased anxietylike behavior in the elevated plus maze, spending more time in the closed arms and less in the center than the controls; (c) increased locomotion in the open field (OF) and novel object (NO) tests; (d) increased startle response, both as the average of the session and in the first pulse; (e) increased prepulse inhibition (PPI); (f) increased depression-like behavior, displaying increased time floating in the forced swimming test and $(\mathbf{g})$ increased fear-related behavior, as indicated by their enhanced freezing after repeated exposure to mild aversive stimulation (a $0.2-\mathrm{mA}$ footshock delivered every $2 \mathrm{~min}$; $\mathrm{F} 0$ ) and by reduced exploration of an unfamiliar male when tested during estrus (F1). F0: $N=7-10$ per group; F1: $N=11-13$ per group. The results are the mean \pm s.e.m. ${ }^{*} P<0.05,{ }^{\star \star} P<0.01$ versus control group.

peripubertally stressed fathers. These rats were more aggressive toward female partners during cohabitation (Figure 2c, Supplementary Tables S6, S7) despite the female showing increased defensive-submissive behavior (Figure 2d, Supplementary Tables S8, S9).

Physiological and behavioral impact of cohabitation with the male offspring of peripubertally stressed males on the female rats. As done with the peripubertally stressed males, we evaluated the impact of the interaction of their male offspring on their female partners after a 21-day cohabitation. Strikingly, the physiological [reduced body weight (Supplementary Figure S1, Supplementary Table S29); reduced plasma corticosterone morning levels (Figure 3a, Supplementary Table S10)] and behavioral [increased anxiety-like behavior and startle responses (Figures $3 b$ and $d$, Supplementary Tables S12, S19), locomotor reactivity (Figure 3c, Supplementary Tables S14, S16-S18), PPI (Figure 3e, Supplementary Table S21), depression-like behavior (Figure $3 f$, Supplementary Table S22) and fear responses (Figure $3 g$, Supplementary Tables
S23, S24)] impacts of cohabitation with the male offspring of peripubertally stressed males on the female partners was essentially a mirror of the alterations displayed by the female partners of the peripubertally stressed males (that is, their mothers).

In order to assess whether the endocrine and behavioral alterations observed in the abused females (from both F0 and F1) were mutually associated, we performed correlational analyses (see Supplementary Table S25 for controls and Supplementary Table S26 for stressed). Whereas some of the correlations were found both in the control and stressed groups (that is, a positive correlation between floating in the forced swim and distance in the OF), other were specifically observed in the stressed females (that is, a negative correlations between PPI and percent time in the closed arms, distance in the OF and floating in the forced swim). Remarkably, some of the correlations observed in control animals between morning corticosterone levels and some behavioral parameters (that is, negative correlations with PPI and locomotor reactivity to the novel object) were not found in the stressed group. 

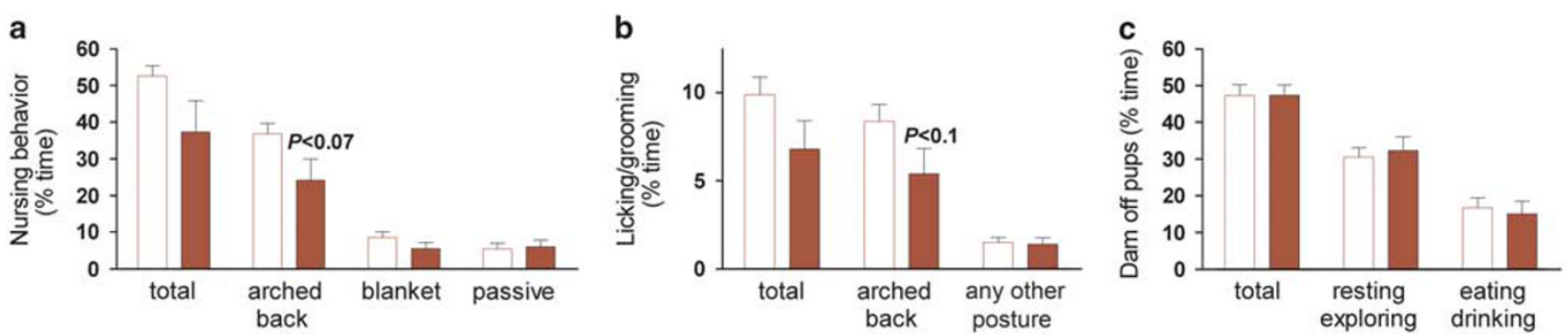

Dams whose offspring are included in F1 study
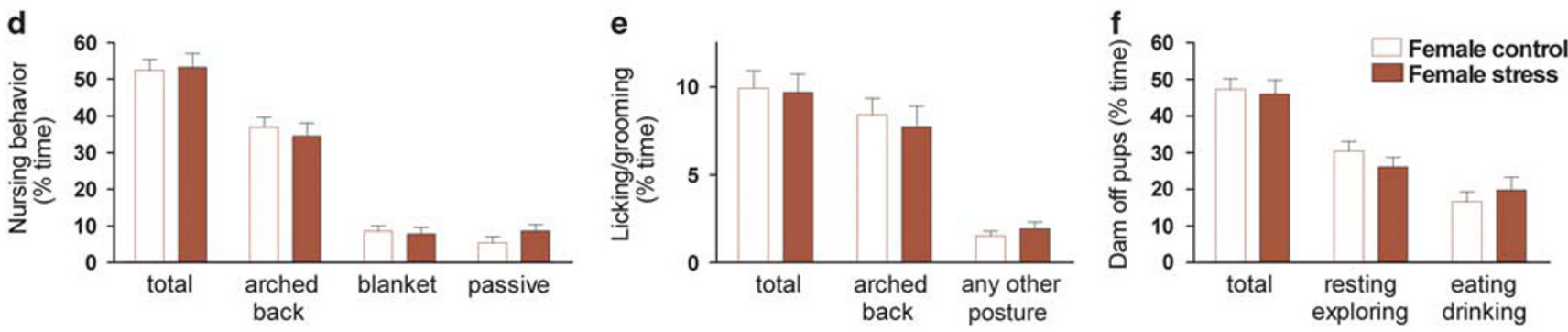

g
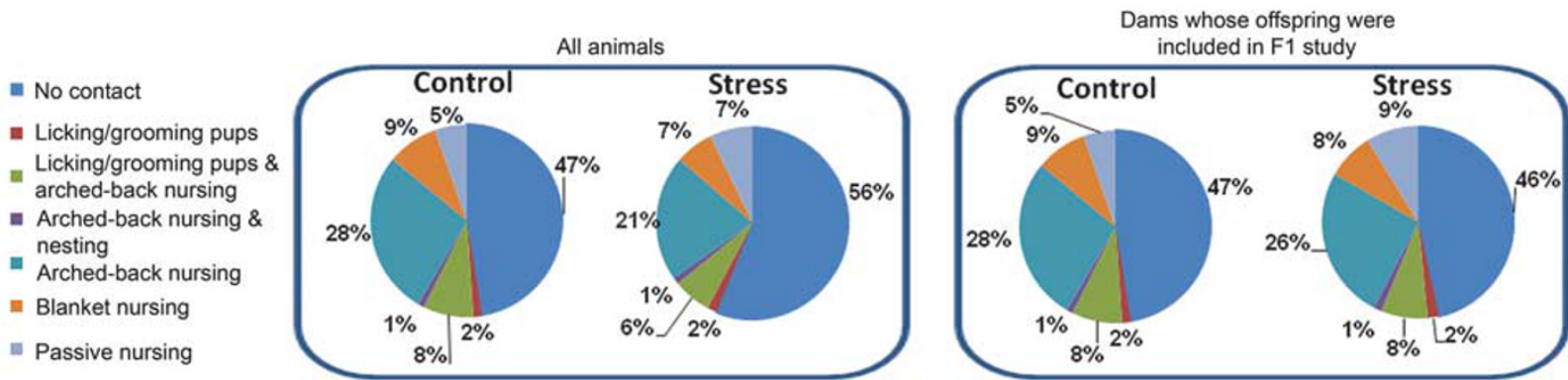

Figure 4 Effects of F0 male-female cohabitation on female maternal behavior during the first 6 days postpartum. See Supplementary Material for descriptions of different types of maternal care measures. (a-c) Analyses including all females in the F0 study indicated that females that cohabited with peripubertally stressed males show a reduced maternal care in certain measures. However, the significance of these tendencies was mainly because of three dams in the stress group that cannibalized their pups. $N=10$ per group. (d-f) Analyses performed excluding the data from the three dams that cannibalized their pups clearly indicate a lack of significant differences between the groups in any of the scored maternal behaviors. The offspring of these dams were included in the F1 study. Control, $N=10 ; S t r e s s, N=7$. The results are the mean \pm s.e.m. (g) Percentage of cumulative time that stress and control dams spent engaging in each category of observed maternal behavior; representing raw data from panels a-f.

Neurobiological impact of cohabitation with the peripubertally stressed males or their male offspring on the female rats. To evaluate the reactivity of serotonergic neurons in the DRN in females previously living with an aggressive male (that is, either the peripubertally stressed males or their offspring), we exposed the females to an unfamiliar male for $15 \mathrm{~min}$ while in diestrus (that is, when female rats are more susceptible to aversive stimuli). Each male and female pair was confined in close proximity within the same fresh home cage. The pair was separated by a transparent plastic wall with holes that allowed smelling and observation but prevented direct physical contact. Two hours after this interaction, brains were collected to evaluate the activation of DRN 5-HT neurons (as assessed by immunohistochemical analyses of protein expression of the immediate early gene c-Fos in 5-HT-labeled neurons (Figure 5b, Supplementary Tables S27)). Additional groups of females for each experimental condition were killed in diestrus under basal conditions. When exposed to an unfamiliar male, the two groups of females that had previously lived with an aggressive male displayed a much greater ( 2.5-fold increase) c-Fos expression in DRN 5-HT neurons than the female rats that had previously been partnered with the control males (Figure 5, Supplementary Tables S28, S31, S32).

\section{Discussion}

We investigated whether male's aggressive behavior toward female partners linked to an early traumatic stress could emerge independently of cultural and social learning factors. We also extended this question to the transmission of this aggressive behavior to the male offspring of the peripubertally stressed fathers. We used a rat model (that is, devoid of human cultural influences) to explore the possibility that 
a
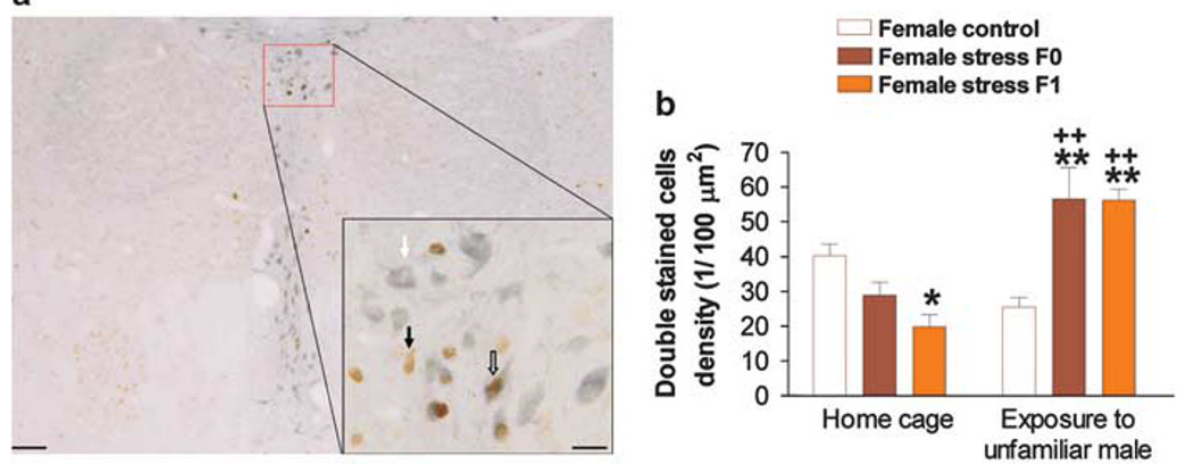

c
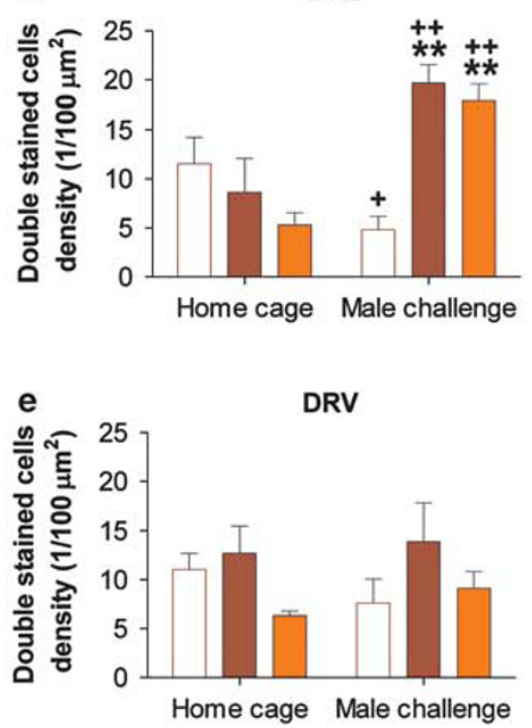

d
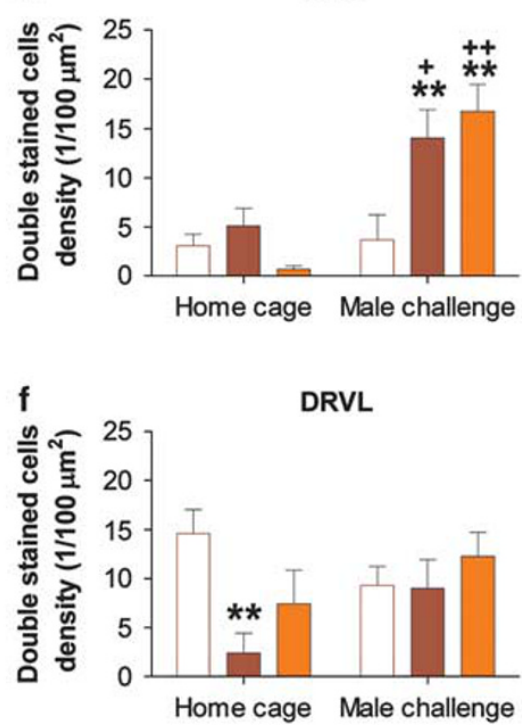

Figure 5 Impact of male-female cohabitation on the percentage of dorsal raphe neurons double-labeled for serotonin (5-HT) and c-Fos. Ten weeks after the end of the male-female cohabitation, females were killed either under basal conditions (home cage; $N=4-5$ per group) or $2 \mathrm{~h}$ after being exposed for 15 min to an unfamiliar male ( $N=6-8$ per group) while in diestrus. (a) Representative photomicrographs showing c-Fos (brown) and 5-HT (grey) immunoreactivity in the dorsal raphe nucleus (DRN), with inset depicting cells single-labeled c-Fos (black arrow), 5-HT (white arrow) or double-labeled (white arrow with black outline). Scale bar $=200 \mu \mathrm{m}$, inset $=20 \mu \mathrm{m}$. (b) When exposed to an unfamiliar male, the percentage of double-labeled 5-HT and c-Fos neurons was enhanced in the F0 and F1 female stress groups relative to the controls. Under basal conditions, the $\mathrm{F} 1$ female stress group showed a reduction in these double-labeled neurons. (c) Results in the dorsal DR (DRD); (d) results in the caudal DR (DRC); (e) Results in the ventral DR (DRV); (f): Results in the ventrolateral DR (DRVL). The results are the mean \pm s.e.m. ${ }^{*} P<0.05$ and ${ }^{\star *} P<0.01$ versus control group under the same conditions. ${ }^{+} P<0.05$ and ${ }^{++} P<0.01$ versus the same group home cage.

biological factors could have an important role in these phenomena. Our results show that peripubertally stressed males (F0) displayed high aggression levels against their female partners. On its turn, female partners of the peripubertally stressed males showed a marked alteration in physiological (decreased body weight and basal plasma corticosterone) and behavioral responses (including increased anxiety- and depression-like behaviors). Strikingly, our results also showed that the male offspring (F1) reproduced the response pattern of their peripubertally stressed fathers. These rats were more aggressive toward female partners during cohabitation despite the female showing increased defensive-submissive behavior. Furthermore, the physiological and behavioral impact of cohabitation with the male offspring of peripubertally stressed males on the female partners was essentially a mirror of the alterations displayed by the female partners of the peripubertally stressed males (that is, their mothers).

In humans, surviving victims of domestic violence typically show long-term negative health consequences, notably including psychopathological alterations. ${ }^{22,23}$ In our rat model, we show that the rat female partners of either peripubertally stressed males or their male offspring displayed decreased body weights both during and up to 10 weeks after cohabitation. These females also showed an altered hypothalamus-pituitary-adrenal axis function, as indicated by alterations in the circadian pattern of plasma corticosterone levels monitored 7 weeks after cohabitation and by enlarged adrenal glands relative to the control females. At the behavioral level, they showed: (i) enhanced anxiety-like behavior in the elevated plus maze and startle responses; (ii) increased motor reactivity both in an OF and in response to a novel object; (iii) increased PPI; (iv) enhanced 
depression-like behavior in the FST and (v) enhanced fear responses. Altogether, these results largely resemble the type of cortisol alterations ${ }^{24}$ and anxious and depressive behavior observed in abused women. ${ }^{25-27}$ Moreover, these symptoms match the alterations observed in women with posttraumatic stress disorder, a syndrome frequently described in abused women. ${ }^{27,28}$ However, a note of caution should be added when considering the constellation of symptoms found in our rat study as a general syndrome. Correlational analyses indicated that the association between some behavioral parameters in stressed females show a similar pattern as in controls (for example, the higher the locomotor reactivity, the higher the floating in the FST). Importantly, they also revealed a specific pattern of associated comorbidity in the stressed females (for example, the higher the anxiety- and depression-like behaviors, the lower the PPI).

To further validate the trauma for the females of interacting with an aggressive male at a neurobiological level, we studied the activation of serotonergic neurons in the DRN. The DRN is a small nucleus located in the brainstem with primarily serotonergic neurons (5-HT). In association with the role of $5-\mathrm{HT}$ in mood modulation, ${ }^{29}$ the serotonergic neurons in the dorsal raphe have been implicated in the pathophysiology of depression, particularly with respect to learned helplessness. This phenomenon is related to behavioral deficits in coping with stressful situations, which is displayed as a consequence of prior exposure to uncontrollable stress. ${ }^{30}$ In rats, these neurons are highly activated by aversive uncontrollable events leading to the development of depression-like behaviors. ${ }^{19,30}$ We found that when exposed to an unknown male, female partner of either peripubertally stressed males or their male offspring displayed an increased activity in DRN serotonergic cells. This result is consistent with a recent report showing increased gene expression of two 5-HTrelated transcription factors in the DRN 5-HT neurons of women, but not men, with major depressive disorder. ${ }^{31}$ Importantly, in our rat study, activation of 5-HT neurons was observed in the dorsal and caudal parts of the DR, regions that target limbic structures, such as hippocampus, septum and medial prefrontal cortex ${ }^{32}$ where the serotonergic system is implicated in the regulation of anxiety. ${ }^{33}$ A question that arises from these findings is whether these alterations in serotonergic reactivity are linked to the described blunting in the morning corticosterone levels and the emotional changes. Although further studies are needed to provide a mechanistic response, support for this hypothesis is suggested by a strong evidence of the existence of a close bidirectional relationship between the hypothalamus-pituitary-adrenal axis and the serotonergic system. ${ }^{34,35}$ More specifically, a link between impaired glucocorticoid function and hyperresponsiveness of the raphe 5-HT system following stress has been reported in genetically modified animals that showed increased reactivity to stress. ${ }^{36}$

The high aggression levels displayed by peripubertally stressed males against their female partners is consistent with human data, indicating that exposure to childhood trauma is especially high in perpetrators of domestic violence. ${ }^{4,5}$ The potential of early life stress (maternal separation from the pups during the early postnatal period) to induce increased inter-male aggression in rats has been previously reported. ${ }^{37}$ To our knowledge, this is the first report showing that early life stress in rats can also lead to increased aggression toward female partners. Thus, our data support the view that biological factors triggered by adverse juvenile experiences can have a central role in the maturation of male perpetrators of intimate domestic violence during adulthood and illustrate the negative consequences experienced by their female partners.

Although most studies of the intergenerational transmission of family violence in humans have focused on the individual's lifespan from victimization to perpetration, there are historical and epidemiological accounts that the transmission of violence can occur across several generations. ${ }^{38,39}$ This concept is further supported by increased cases of intimate partner violence among children of surviving victims of domestic violence. ${ }^{40}$ Thus, our data showing increased aggressive behavior toward females in the peripubertally stressed rats' offspring fits with the human literature, illustrating the transmission of violence toward females across generations and further support a role for biological factors in this phenomenon.

The observation that male offspring displaying increased aggression toward their female partners is consistent with emerging evidence supporting the occurrence of paternal effects in offspring in the absence of postnatal fatheroffspring interaction. ${ }^{41}$ Multiple mechanisms may be driving these paternal effects, ${ }^{41}$ including the impact of gestational ${ }^{42}$ or early postnatal stress associated with maternal anxiety, ${ }^{43}$ changes in the quality of the maternal care ${ }^{44}$ [however, it is important to note that no differences in maternal care were observed among the offspring of the control and peripubertally stressed males used in the F1 study (Figure 4 and Supplementary Figure S2)] or epigenetic factors transmitted through the germline. ${ }^{45}$ Rodent studies have presented evidence for the transmission of behavioral traits across generations through both maternal behavioral effects ${ }^{44,46}$ and through paternal mechanisms involving epigenetic transmission through the germline ${ }^{47,48}$ or through other paternallyinduced maternal adaptations. ${ }^{49}$

Altogether, our results highlight a key role for biological factors in the link between juvenile exposure to trauma and the ensuing development of pathological aggression toward intimate partners. These findings, along with the assemblage of physiological and behavioral symptoms observed in females cohabiting with either the peripubertally stressed males or with their male offspring, confirm the detrimental impact of these close interactions on females' indexes of physical and mental health. Our results also indicate that this phenotype can be transmitted to the next generation in the absence of father-offspring postnatal interaction. Although extrapolation from animals to humans should always be made with caution, our work strongly supports the need to extend the currently dominant psychosocial explanations of the traumatic stress-induced violence cycle to include biological factors. In addition to opening new perspectives for prevention and intervention programs addressing the inter- and trans-generational transmission of intimate partner violence, our findings urge the development of research efforts aimed at identifying the precise biological mechanisms driving these effects. 


\section{Conflict of interest}

The authors declare no conflict of interest.

Acknowledgements. We thank Jocelyn Grosse, Coralie Siegmund, Vanessa Singh, Fabian Jordi and Muy-Cheng Peich for their excellent technical assistance. This work was supported by grants from the Swiss National Science Foundation (310000-120791 and 31003AB-135710; Sinergia CRSIK3-122691; and the NCCR 'The synaptic basis of mental diseases') and intramural funding from the EPFL.

1. Butchart A, Garcia-Moreno C, Mikton C. Preventing intimate partner and sexual violence against women: taking action and generating evidence. World Health Organization: Geneva, Switzerland, 2010.

2. Ellsberg $M$, Jansen $H A$, Heise $L$, Watts $\mathrm{CH}$, Garcia-Moreno $\mathrm{C}$. Intimate partner violence and women's physical and mental health in the WHO multi-country study on women's health and domestic violence: an observational study. Lancet 2008; 371: 1165-1172.

3. Taft AJ, Hegarty KL. Intimate partner violence against women: what outcomes are meaningful? JAMA 2010; 304: 577-579.

4. Heise LL. Violence against women: an integrated, ecological framework. Violence Against Women 1998; 4: 262-290.

5. McKinney CM, Caetano R, Ramisetty-Mikler S, Nelson S. Childhood family violence and perpetration and victimization of intimate partner violence: findings from a national population-based study of couples. Ann Epidemiol 2009; 19: 25-32.

6. Caspi A, McClay J, Moffitt TE, Mill J, Martin J, Craig IW et al. Role of genotype in the cycle of violence in maltreated children. Science 2002; 297: 851-854.

7. McGowan PO, Sasaki A, D'Alessio AC, Dymov S, Labonte B, Szyf M et al. Epigenetic regulation of the glucocorticoid receptor in human brain associates with childhood abuse. Nat Neurosci 2009; 12: 342-348.

8. McCrory E, De Brito SA, Viding E. The impact of childhood maltreatment: a review of neurobiological and genetic factors. Front Psychiatry 2011; 2: 48

9. Pinto LA, Sullivan EL, Rosenbaum A, Wyngarden N, Umhau JC, Miller MW et al. Biological correlates of intimate partner violence perpetration. Aggr Viol Behav 2010; 15: 387-398.

10. Israel $E$, Stover $C$. Intimate partner violence: the role of the relationship between perpetrators and children who witness violence. J Interpers Violence 2009; 24: 1755-1764.

11. Widom CS, Brzustowicz LM. MAOA and the "cycle of violence:" childhood abuse and neglect, MAOA genotype, and risk for violent and antisocial behavior. Biol Psychiatry 2006; 60: 684-689.

12. George DT, Phillips MJ, Doty L, Umhau JC, Rawlings RR. A model linking biology, behavior and psychiatric diagnoses in perpetrators of domestic violence. Med Hypotheses 2006; 67 : 345-353.

13. Fergusson DM, Boden JM, Horwood LJ, Miller A, Kennedy MA. Moderating role of the MAOA genotype in antisocial behaviour. Br J Psychiatry 2012; 200: 116-123

14. George DT, Phillips MJ, Lifshitz M, Lionetti TA, Spero DE, Ghassemzedeh N et al. Fluoxetine treatment of alcoholic perpetrators of domestic violence: a 12-week, doubleblind, randomized, placebo-controlled intervention study. J Clin Psychiatry 2011; 72: $60-65$.

15. Moore BC, Easton CJ, McMahon TJ. Drug abuse and intimate partner violence: a comparative study of opioid-dependent fathers. Am J Orthopsychiatry 2011; 81: 218-227.

16. Marquez C, Poirier GL, Cordero Ml, Larsen MH, Groner A, Marquis J et al. Abnormal aggression induced by early life trauma is associated with increased prefrontal MAOA gene expression and epigenetic regulation. PNAS (in review).

17. Toledo-Rodriguez M, Sandi C. Stress during adolescence increases novelty seeking and risk taking behavior in male and female rats. Front Behav Neurosci 2011; 7: 5-17.

18. Champagne FA, Francis DD, Mar A, Meaney MJ. Variations in maternal care in the rat as a mediating influence for the effects of environment on development. Physiol Behav 2003; 79: 359-371.

19. Amat J, Baratta MV, Paul E, Bland ST, Watkins LR, Maier SF. Medial prefrontal cortex determines how stressor controllability affects behavior and dorsal raphe nucleus. Nat Neurosci 2005; 8: 365-371.

20. Mandl AM. The phases of the oestrous cycle in the adult white rat. J Exp Biol 1951; 28: 576-584.

21. Paxinos G, Watson C. The Rat Brain in Stereotaxic Coordinates (Fourth Edition) Academic Press: San Diego, USA, 1998.

22. Campbell JC. Health consequences of intimate partner violence. Lancet 2002; 359: 1331-1336.

23. Helfrich CA, Fujiura GT, Rutkowski-Kmitta V. Mental health disorders and functioning of women in domestic violence shelters. J Interpers Violence 2008; 23: 437-453.

24. Griffin MG, Rescik PA, Yehuda R. Enhanced cortisol suppression following dexamethasone administration in domestic violence survivors. Am J Psychiatry 2005; 162 1192-1199.

25. Stein MB, Kennedy C. Major depressive and post-traumatic stress disorder comorbidity in female victims of intimate partner violence. J Affect Disord 2001; 66: 133-138.

26. Lang AJ, Kennedy CM, Stein MB. Anxiety sensitivity and PTSD among female victims of intimate partner violence. Depress Anxiety 2002; 16: 77-83.

27. Pico-Alfonso MA, Garcia-Linares MI, Celda-Navarro N, Blasco-Ros C, Echeburua E, Martinez M. The impact of physical, psychological, and sexual intimate male partner violence on women's mental health: depressive symptoms, posttraumatic stress disorder, state anxiety, and suicide. J Womens Health 2006; 15: 599-611.

28. Dutton MA, Green BL, Kaltman SI, Roesch DM, Zeffiro TA, Krause ED. Intimate partner violence, PTSD, and adverse health outcomes. J Interpers Violence 2006; 21: 955-968.

29. Hariri AR, Holmes A. Genetics of emotional regulation: the role of the serotonin transporter in neural function. Trends Cogn Sci 2006; 10: 182-191.

30. Maier SF, Watkins LR. Stressor controllability and learned helplessness: the roles of the dorsal raphe nucleus, serotonin, and corticotropin-releasing factor. Neurosci Biobehav Rev 2005; 29: 829-841.

31. Goswami DB, May WL, Stockmeier CA, Austin MC. Transcriptional expression of serotonergic regulators in laser-captured microdissected dorsal raphe neurons of subjects with major depressive disorder: sex-specific differences. J Neurochem 2010; 112: 397-409.

32. Kohler $\mathrm{C}$, Steinbusch $\mathrm{H}$. Identification of serotonin and non-serotonin-containing neurons of the mid-brain raphe projecting to the entorhinal area and the hippocampal formation. A combined immunohistochemical and fluorescent retrograde tracing study in the rat brain. Neurosci 1982; 7: 951-975.

33. Abrams JK, Johnson PL, Hay-Schmidt A, Mikkelsen JD, Shekhar A, Lowry CA. Serotonergic systems associated with arousal and vigilance behaviors following administration of anxiogenic drugs. Neurosci 2005; 133: 983-997.

34. Meijer OC, de Kloet ER. Corticosterone and serotonergic neurotransmission in the hippocampus: functional implications of central corticosteroid receptor diversity. Crit Rev Neurobiol 1998; 12: 1-20.

35. Howell BR, Sanchez MM. Understanding behavioral effects of early life stress using the reactive scope and allostatic load models. Dev Psychopathol 2011; 23: 1001-1016.

36. Linthorst AC, Flachskamm C, Barden N, Holsboer F, Reul JM. Glucocorticoid receptor impairment alters CNS responses to a psychological stressor: an in vivo microdialysis study in transgenic mice. Eur J Neurosci 2000; 12: 283-291.

37. Veenema AH, Blume A, Niederle D, Buwalda B, Neumann ID. Effects of early life stress on adult male aggression and hypothalamic vasopressin and serotonin. Eur J Neurosci 2006; 24: $1711-1720$

38. Doumas D, Margolin G, John RS. The intergenerational transmission of aggression across three generations. J Fam Violence 1994; 9: 157-175.

39. Atkinson J, Nelson J, Atkinson C. Trauma, transgenerational transfer and effects on community wellbeing. In: Purdie M, Dudgeon P, Walker R (eds). Working Together: Aboriginal and Torres Strait Islander Mental Health and Wellbeing Principles and Practice. Commonwealth of Australia, 2010, pp 135-144.

40. Roberts AL, Gilman SE, Futzmaurice G, Decker MR, Koenen KC. Witness of intimate partner violence in childhood and perpetration of intimate partner violence in adulthood. Epidemiology 2010; 21: 809-818.

41. Curley JP, Mashoodh R, Champagne FA. Epigenetics and the origins of paternal effects. Horm Behav 2011; 59: 306-314

42. Radtke KM, Ruf M, Gunter HM, Dohrmann K, Schauer M, Meyer A, Elbert T. Transgenerational impact of intimate partner violence on methylation in the promoter of the glucocorticoid receptor. Transl Psychiatry 20111: e21; doi:10.1038/tp.2011.21.

43. Korosi A, Baram TZ. The pathways from mother's love to baby's future. Front Behav Neurosci 2009; 3, 10.3389/neuro.08.027.2009.

44. Champagne FA, Meaney MJ. Stress during gestation alters postpartum maternal care and the development of the offspring in a rodent model. Biol Psychiatry 2006; 59: 1227-1235.

45. Franklin TB, Mansuy IM. Epigenetic inheritance in mammals: evidence for the impact of adverse environmental effects. Neurobiol Dis 2009; 39: 61-65.

46. Francis D, Diorio J, Liu D, Meaney MJ. Nongenomic transmission across generations of maternal behavior and stress responses in the rat. Science 1999; 286: 1155-1158.

47. Franklin TB, Russig H, Weiss IC, Graff J, Linder N, Michalon A et al. Epigenetic transmission of the impact of early stress across generations. Biol Psychiatry 2010; 68: 408-415.

48. Morgan $\mathrm{CP}$, Bale TL. Early prenatal stress epigenetically programs dysmasculinization in second-generation offspring via the paternal lineage. J Neurosci 2011; 31: 11748-11755.

49. Dietz DM, LaPlant Q, Watts EL, Hodes GE, Russo SJ, Feng J et al. Paternal transmission of stress-induced pathologies. Biol Psychiatry 2011; 70: 408-414.

(c)

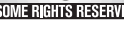

Translational Psychiatry is an open-access journal published by Nature Publishing Group. This work is licensed under the Creative Commons Attribution-Noncommercial-No Derivative Works 3.0 Unported License. To view a copy of this license, visit http://creativecommons.org/licenses/by-nc-nd/3.0/ 